\title{
Effect of levodopa on frontal-subcortical and posterior cortical functioning in patients with Parkinson's disease
}

Amara $\underline{\mathrm{Gul}}^{1}, \mathrm{PhD}$, Javed $\underline{\text { Yousaf }}^{1}, \mathrm{MSc}$

INTRODUCTION Parkinson's disease (PD) is associated with cognitive decline but little is known about frontal-subcortical and posterior cortical cognitive functioning in patients with PD. The present study was designed to: (a) compare frontal and posterior cognitive functioning between patients with PD and healthy controls; (b) determine the effect of levodopa (L-dopa) on frontal-subcortical and posterior cortical cognitive functions; and (c) identify predictors of cognitive functions in patients with PD.

METHODS 50 patients diagnosed with PD from April 2016 to May 2017 at Civil Hospital, Bahawal Victoria Hospital, Bahawalpur, and Nishter Hospital Multan, Pakistan, and 50 healthy individuals from the community participated in our study. Patients had two testing sessions - first, at the time of diagnosis before taking L-dopa medication to determine baseline scores; and second, after at least three months of L-dopa medication. Participants completed the Parkinson's Disease-Cognitive Rating Scale.

RESULTS Patients with PD showed impaired performance on frontal-subcortical and posterior cortical functions in contrast with the control group. L-dopa medication had beneficial effects on frontal-subcortical and posterior cortical functioning in patients with PD. Disease duration was a significant predictor of cognitive performance in patients with PD. CONCLUSION L-dopa medication improves frontal-subcortical and posterior cortical cognitive functioning in patients with PD. Disease duration is a marker of cognitive decline in PD.

Keywords: cognition, frontal-subcortical functions, levodopa, Parkinson's disease, posterior cortical functions

\section{INTRODUCTION}

Parkinson's disease (PD) is one of the most common neurodegenerative disorders that results from loss of neurons in the nigrostriatal pathway, which reduces dopamine levels in the striatum. ${ }^{(1)}$ Depletion of dopaminergic neurons is associated with the frontostriatal circuit, which modulates cognitive, motor and affective processes. ${ }^{(2)}$ Specifically, this circuit is involved in executive functions required to initiate goal-directed behaviour and conflict resolution. ${ }^{(3)}$ Cortical areas in the frontal lobe, including the dorsolateral prefrontal cortex and orbitofrontal cortices, are involved in information processing, rule-based learning, working memory, attention and planning. ${ }^{(4)}$ Frontal cortical regions provide top-down regulation of attention, inhibition and cognitive control through connections with the posterior subcortical structures. These structures are sensitive to changes in the levels of neurotransmitters. Thus, brain functions get altered, but this can be improved by medication. ${ }^{(5)}$ Decreased levels of dopamine in the brain can cause tremors, muscle rigidity and impaired reflexes. ${ }^{(6)}$ These symptoms can be well controlled by levodopa (L-dopa), which is a standard treatment for PD. ${ }^{(7)}$

$\mathrm{PD}$ is associated with decline across several cognitive functions, such as attention, memory and task switching, due to striatal dopaminergic depletion and global brain volume. ${ }^{(8,9)}$ The dopamine system is involved in cognitive decline associated with PD. This has been observed in the form of reduced 18 fluorodopa uptake in the frontal cortex and caudate nucleus in patients with PD during the performance of neuropsychological tests. ${ }^{(10)}$ It has been observed that three-month L-dopa treatment for patients with PD has beneficial effects on their neuropsychological performance. ${ }^{(11)}$ There is an increase in dopaminergic burst and excitatory activity in the direct pathway and inhibition of activity in the indirect pathway of the cortico-striatal-thalamo-cortical loop with L-dopa medication. ${ }^{(12)}$ As a result, irrelevant responses are suppressed and cognitive functions are improved. ${ }^{(13-15)}$

In this study, we aimed to: (a) compare frontal-subcortical and posterior cortical functioning between patients with PD and healthy individuals; (b) clarify the effects of L-dopa treatment on frontal-subcortical and posterior cortical functions in patients with PD; and (c) identify predictors of cognitive performance in patients with PD. We hypothesised or expected that: (a) patients with PD would show impaired performance on frontal-subcortical and posterior cortical functioning in contrast with healthy individuals; (b) L-dopa medication would have beneficial effects on frontalsubcortical and posterior cortical functioning in patients with PD; and (c) duration of disease would be a significant predictor of cognitive performance for patients with PD.

\section{METHODS}

This study was approved by the board of studies at The Islamia University of Bahawalpur, Pakistan. 50 patients diagnosed with idiopathic PD participated in the study from April 2016 to May 2017 at Civil Hospital, Bahawal Victoria Hospital, Bahawalpur, and Nishter Hospital Multan, Pakistan. Patients were tested twice to compare the performance of frontal-subcortical and posterior cortical cognitive functions before and after taking L-dopa medication: (a) at the time of diagnosis when they were

${ }^{1}$ Department of Applied Psychology, The Islamia University of Bahawalpur, Pakistan

Correspondence: Dr Amara Gul, Assistant Professor, Department of Applied Psychology, The Islamia University of Bahawalpur, Pakistan. amara_psychology@hotmail.com 
Table I. Demographic and clinical characteristics.

\begin{tabular}{|c|c|c|c|c|}
\hline \multirow[t]{2}{*}{ Variable } & \multicolumn{2}{|c|}{ Mean \pm SD (range)/No. } & \multirow[t]{2}{*}{$t$ (df) } & \multirow[t]{2}{*}{ p-value } \\
\hline & Control group $(n=50)$ & Patients with PD $(n=50)$ & & \\
\hline Age (yr) & $55.78 \pm 5.78(43-65)$ & $57.02 \pm 5.29(45-65)$ & $1.15(49)$ & 0.25 \\
\hline \multicolumn{5}{|l|}{ Gender } \\
\hline Men & 25 & 25 & & \\
\hline Women & 25 & 25 & & \\
\hline Disease duration (yr) & & $3.50 \pm 1.31(2-5)$ & & \\
\hline UPDRS activities of daily living subscale score & & $29.34 \pm 3.09(24-35)$ & & \\
\hline Daily L-dopa dose (mg) & & $483.94 \pm 59.31$ & & \\
\hline
\end{tabular}

L-dopa: levodopa; PD: Parkinson's disease; SD: standard deviation; UPDRS: Unified Parkinson's Disease Rating Scale

not taking any medication for PD; and (b) when patients were stable and on daily L-dopa medication for at least three months.

The exclusion criteria for patients with PD were as follows: (a) intake of any medication except L-dopa preparation; (b) presence of symptomology or previous medical history of any neurological disorder except PD (e.g. stroke and head injury); (c) presence of symptoms or history of any psychiatric disorder screened through the Mini International Neuropsychiatric Interview (MINI);(16) and (d) dementia, as screened through the Mini-Mental Parkinson, with cut-off scores $\leq 17$ out of $32 .{ }^{(17)}$

Demographically matched, healthy individuals from the local community participated in the study as the control group $(n=50)$. The exclusion criteria for the control group were as follows: (a) intake of any medication; (b) presence or previous medical history of any neurological disorder; (c) presence of symptoms or history of any psychiatric disorder, as screened through the $\mathrm{MINI}$; ${ }^{(16)}$ and (d) dementia, as screened through the Mini Mental State Examination (MMSE), with cut-off scores $\leq 24{ }^{(18)}$

Participants gave written informed consent. They were administered the 'activities of daily living' subscale of the Unified Parkinson's Disease Rating Scale ${ }^{(19)}$ and Parkinson's DiseaseCognitive Rating Scale (PD-CRS). ${ }^{(20)}$ This scale helps in the assessment of frontal-subcortical and posterior cortical cognitive functions pertaining to neural correlates. The frontal-subcortical functions include attention, clock-drawing, working memory, alternating verbal memory (immediate/delayed) and action verbal fluency, whereas posterior cortical cognitive functions are confrontation naming and copying a drawing of a clock. The cumulative score of subtests corresponded to scores in the range 0-114 for frontal-subcortical and 0-20 for posterior cortical cognitive functions; higher scores represented better performance. This scale has good reliability and validity. Measurement of cognitive functions was randomised across participants to avoid researcher bias. Following completion of the study, all participants were thanked for taking part in it.

For statistical analyses, demographic and clinical characteristics were examined using descriptive statistics and Student's $t$-test. Repeated-measures analysis of variance (ANOVA) was used to compare performance on each subscale of PDCRS, with factor of 3 (control group vs. patients with PD before L-dopa medication at baseline as compared to patients with PD after three-month L-dopa medication). Individual ANOVAs were conducted to assess frontal-subcortical scores and posterior cortical scores before and after L-dopa treatment with factor of 2 for treatment (patients with PD before L-dopa medication at baseline as compared to patients with PD after three-month L-dopa medication). Regression analysis was conducted using the total scores of patients with PD on PD-CRS as a dependent variable and their clinical characteristics (e.g. age, gender and duration of disease) as independent variables to identify predictors of cognitive performance for patients with PD.

\section{RESULTS}

The overall demographic and clinical characteristics of participants are shown in Table I. Our results indicated the presence of cognitive impairment in patients with $\mathrm{PD}$ when compared with the control group for cortical and subcortical cognitive functions - immediate free recall verbal memory $[F(2,98)=810.51, \mathrm{p}<0.001, \eta \mathrm{p} 2=0.94] ;$ confrontation naming $[F(2,98)=886.17, \mathrm{p}<0.001, \eta \mathrm{p} 2=0.94]$; sustained attention $[F(2,98)=810.21, \mathrm{p}<0.001, \eta \mathrm{p} 2=0.94]$; working memory $[F(2,98)=1,088.67, \mathrm{p}<0.001, \eta \mathrm{p} 2=0.95] ;$ unprompted drawing of a clock $[F(2,98)=1,303.89, \mathrm{p}<0.001, \eta \mathrm{p} 2=0.96]$; alternating verbal fluency $[F(2,98)=1,737.02, \mathrm{p}<0.001, \eta p 2=0.97]$; and action verbal fluency $[F(2,98)=1,951.67, \mathrm{p}<0.001$, $\eta p 2=0.97]$. The mean scores, provided in Table II, showed that cognitive performance of patients with PD improved after L-dopa medication. Although cognitive deficits existed in patients with PD, in contrast with the control group, their scores showed significant improvement in all cognitive domains irrespective of cortical or subcortical functioning following L-dopa medication for three months.

Results of separate ANOVAs conducted to examine the effects of treatment on each cognitive function among patients with PD showed the significant effect of L-dopa medication, with improvement of cognitive functioning after treatment - immediate free recall verbal memory $[F(1,49)=405.87$, $\mathrm{p}<0.001, \eta \mathrm{p} 2=0.89]$; confrontation naming $[F(1,49)=314.71$, $\mathrm{p}<0.001, \eta \mathrm{p} 2=0.86]$; sustained attention $[F(1,49)=365.18$, $\mathrm{p}<0.001, \eta \mathrm{p} 2=0.88]$; working memory $[F(1,49)=492.07$, $\mathrm{p}<0.001, \eta p 2=0.90]$; unprompted drawing of a clock $[F(1,49)=355.64, \mathrm{p}<0.001, \eta \mathrm{p} 2=0.87]$; alternating verbal fluency $[F(1,49)=265.35, \mathrm{p}<0.001, \eta p 2=0.84]$; and action verbal fluency $[F(1,49)=365.18, \mathrm{p}<0.001, \eta \mathrm{p} 2=0.88]$. 
Table II. Mean scores for the subscales of PD-CRS.

\begin{tabular}{|c|c|c|}
\hline \multirow[t]{2}{*}{ Subscale } & \multicolumn{2}{|c|}{ Score } \\
\hline & Mean \pm SD & $95 \% \mathrm{Cl}$ \\
\hline \multicolumn{3}{|c|}{$\begin{array}{l}\text { Immediate free recall verbal } \\
\text { memory }\end{array}$} \\
\hline Control group & $10.98 \pm 0.84$ & $10.74-11.22$ \\
\hline \multicolumn{3}{|l|}{ Patients with PD } \\
\hline At baseline & $5.00 \pm 0.78$ & $4.77-5.22$ \\
\hline After L-dopa medication & $7.58 \pm 0.49$ & $7.43-7.72$ \\
\hline \multicolumn{3}{|l|}{ Confrontation naming } \\
\hline Control group & $18.92 \pm 1.50$ & $18.49-13.34$ \\
\hline \multicolumn{3}{|l|}{ Patients with PD } \\
\hline At baseline & $5.42 \pm 1.62$ & $4.95-5.88$ \\
\hline After L-dopa medication & $11.66 \pm 1.74$ & $11.16-12.15$ \\
\hline \multicolumn{3}{|l|}{ Sustained attention } \\
\hline Control group & $9.44 \pm 0.50$ & $9.29-9.58$ \\
\hline \multicolumn{3}{|l|}{ Patients with PD } \\
\hline At baseline & $3.90 \pm 0.78$ & $3.67-4.12$ \\
\hline After L-dopa medication & $6.96 \pm 0.69$ & $6.76-7.15$ \\
\hline \multicolumn{3}{|l|}{ Working memory } \\
\hline Control group & $9.34 \pm 0.47$ & $9.20-9.47$ \\
\hline \multicolumn{3}{|l|}{ Patients with PD } \\
\hline At baseline & $2.96 \pm 0.80$ & $2.73-3.18$ \\
\hline After L-dopa medication & $6.28 \pm 0.70$ & $6.08-6.47$ \\
\hline \multicolumn{3}{|c|}{ Unprompted drawing of a clock } \\
\hline Control group & $9.56 \pm 0.50$ & $9.41-9.70$ \\
\hline \multicolumn{3}{|l|}{ Patients with PD } \\
\hline At baseline & $1.92 \pm 0.77$ & $1.69-2.14$ \\
\hline After L-dopa medication & $4.92 \pm 0.85$ & $4.67-5.16$ \\
\hline \multicolumn{3}{|c|}{ Delayed free recall verbal memory } \\
\hline Control group & $10.92 \pm 0.87$ & $10.67-11.66$ \\
\hline \multicolumn{3}{|l|}{ Patients with PD } \\
\hline At baseline & $3.48 \pm 1.12$ & $3.15-3.80$ \\
\hline After L-dopa medication & $6.90 \pm 0.78$ & $6.67-7.12$ \\
\hline \multicolumn{3}{|l|}{ Alternating verbal fluency } \\
\hline Control group & $19.02 \pm 0.79$ & $18.79-19.24$ \\
\hline \multicolumn{3}{|l|}{ Patients with PD } \\
\hline At baseline & $4.08 \pm 1.30$ & $3.70-4.45$ \\
\hline After L-dopa medication & $9.06 \pm 1.53$ & $8.62-9.49$ \\
\hline \multicolumn{3}{|l|}{ Action verbal fluency } \\
\hline Control group & $28.74 \pm 1.02$ & $28.44-29.03$ \\
\hline \multicolumn{3}{|l|}{ Patients with PD } \\
\hline At baseline & $13.26 \pm 1.39$ & $12.86-13.65$ \\
\hline After L-dopa medication & $18.14 \pm 1.38$ & $17.74-18.53$ \\
\hline
\end{tabular}

$\mathrm{Cl}$ : confidence interval; L-dopa: levodopa; PD: Parkinson's disease; PD-CRS: Parkinson's Disease-Cognitive Rating Scale; SD: standard deviation

Repeated-measures ANOVA to examine the effects of treatment on frontal-subcortical functioning showed significant improvement in frontal-subcortical scores after L-dopa medication among patients with PD $[F(1,49)=2,389.74, \mathrm{p}<0.001$, $\eta p 2=0.98]$; mean baseline scores were $34.60 \pm 2.98$, while scores after L-dopa treatment were $59.84 \pm 2.80$. There was also a significant difference between posterior cortical functioning of patients with PD before and after taking L-dopa medication $[F(1,49)=314.71, \mathrm{p}<0.001, \eta \mathrm{p} 2=0.86]$; baseline scores were $5.42 \pm 1.62$, while scores after L-dopa treatment were $11.66 \pm$ 1.74. Regression analysis showed that the duration of disease was a significant predictor of cognitive performance for patients with PD on PD-CRS $\left[\mathrm{R}^{2}=0.51, F(3,49)=16.40, \beta=-0.70\right.$, $\mathrm{p}<0.001 ; \mathrm{t}=6.80, \mathrm{p}<0.001]$, whereas age $(\beta=0.20, \mathrm{t}=1.97$, $\mathrm{p}=0.055)$ and gender $(\beta=-0.04, \mathrm{t}=-0.45, \mathrm{p}=0.649)$ failed to reach levels of significance.

\section{DISCUSSION}

In our study, patients with PD showed impaired performance, in contrast with the control group, on frontal-subcortical and posterior cortical cognitive functions at the time of diagnosis when patients were not taking any medication. PD is associated with decline across several cognitive functions, such as attention, memory and task-switching, due to striatal dopaminergic depletion and global brain volume..$^{(8,9)}$ Cognitive deficits observed in patients with PD are similar to those associated with lesions of the prefrontal cortex. Circuits of the frontal cortex and basal ganglia are dependent upon levels of dopamine. Change in dopamine stimulation alters performance of the frontal cognitive functions. (21) Our results demonstrated that both frontalsubcortical and posterior cognitive functions were affected by dopaminergic depletion.

Among patients with PD, improvements were seen for several cognitive functions pertaining to frontal-subcortical functioning after L-dopa treatment. These cognitive functions included immediate free recall verbal memory, sustained attention, working memory, unprompted drawing of a clock, delayed free recall verbal memory, alternating verbal fluency and action verbal fluency. Likewise, L-dopa treatment improved performance on posterior cortical functioning in our study, as assessed through confrontation naming. Longitudinal studies have shown that dopamine transmission is critical for frontal cortical functions and the administration of L-dopa leads to improvements in cognitive dysfunctions in PD. ${ }^{(22)}$ Previous studies have found that the corticostriatal circuits connecting both frontal and posterior regions to the dorsal caudate nucleus are dopamine-depleted in PD and dopaminergic medication improves performance on cognitive tasks underlying these circuits. ${ }^{(23)}$

Duration of disease was also found to be a significant predictor of the performance of frontal-subcortical and posterior cortical cognitive functions for patients with PD. This result was consistent with the findings of previous studies that have found that neuropsychological performance of patients with PD deteriorates as disease duration increases. ${ }^{(24,25)}$

To the best of our knowledge, this is the first study examining the effect of L-dopa on frontal-subcortical and posterior cortical cognitive functions as well as the various predictors of cognitive performance among patients with PD. Our findings would support clinical practice by helping in the early identification and treatment of cognitive decline in patients with PD.

We conclude that three-month L-dopa medication for patients with PD has beneficial effects for their frontal-subcortical and 
posterior cortical functions. Future studies should examine followup data of patients with PD to ascertain whether improvements seen in their frontal-subcortical and posterior cortical functions after L-dopa treatment is maintained several years after patients are taken off the medication.

\section{REFERENCES}

1. Yeterian EH, Pandya DN. Prefrontostriatal connections in relation to cortical architectonic organization in rhesus monkeys. J Comp Neurol 1991; 312:43-67.

2. Chudasama $Y$, Robbins TW. Functions of frontostriatal systems in cognition comparative neuropsychopharmacological studies in rats, monkeys and humans. Biol Psychol 2006; 73:19-38.

3. Miyake A, Friedman NP. The nature and organization of individual differences in executive functions: four general conclusions. Curr Dir Psychol Sci 2012; 21:8-14

4. Bonelli RM, Cummings JL. Frontal-subcortical circuitry and behavior. Dialogues Clin Neurosci 2007; 9:141-51.

5. Arnsten AF, Rubia K. Neurobiological circuits regulating attention, cognitive control, motivation, and emotion: disruptions in neurodevelopmental psychiatric disorders. J Am Acad Child Adolesc Psychiatry 2012; 51:356-67.

6. Lai BC, Tsui JK. Epidemiology of Parkinson's disease. BCMJ 2001; 43:133-7.

7. Chen SY, Tsai ST. The epidemiology of Parkinson's disease. Tzu Chi Med J 2010; 22:73-83.

8. Narme $\mathrm{P}$, Mouras $\mathrm{H}$, Roussel $\mathrm{M}$, et al. Emotional and cognitive social processes are impaired in Parkinson's disease and are related to behavioral disorders. Neuropsychology 2013; 27:182-92.

9. Jokinen P, Brück A, Aalto S, et al. Impaired cognitive performance in Parkinson's disease is related to caudate dopaminergic hypofunction and hippocampal atrophy. Parkinsonism Relat Disord 2009; 15:88-93.

10. Rinne JO, Portin R, Ruottinen $\mathrm{H}$, et al. Cognitive impairment and the brain dopaminergic system in Parkinson disease: [18F] fluorodopa positron emission tomographic study. Arch Neurol 2000; 57:470-5

11. Molloy SA, Rowan EN, O'Brien JT, et al. Effect of levodopa on cognitive function in Parkinson's disease with and without dementia and dementia with Lewy bodies. J Neurol Neurosurg Psychiatry 2006; 77:1323-8.

12. Frank MJ, Samanta J, Moustafa AA, Sherman SJ. Hold your horses: impulsivity, deep brain stimulation, and medication in parkinsonism. Science 2007;
318:1309-12.

13. Mattis PJ, Tang CC, Ma Y, Dhawan V, Eidelberg D. Networks correlates of the cognitive response to levodopa in Parkinson disease. Neurology 2011; 77:858-65.

14. Jahanshahi M, Wilkinson L, Gahir H, Dharmaindra A, Lagnado DA. Medication impairs probabilistic classification learning in Parkinson's disease. Neuropsychologia 2010; 48:1096-103.

15. Hanna-Pladdy B, Heilman KM. Dopaminergic modulation of the planning phase of skill acquisition in Parkinson's disease. Neurocase 2010; 16:182-90.

16. Sheehan DV, Lecrubier $\mathrm{Y}$, Sheehan $\mathrm{KH}$, et al. The Mini International Neuropsychiatric Interview (M.I.N.I.): the development and validation of a structured diagnostic psychiatric interview for DSM-IV and ICD-10. J Clin Psychiatry 1998; 59:22-33.

17. Mahieux F, Michelet D, Manifacier MJ, et al. Mini-Mental Parkinson: first validation study of a new bedside test constructed for Parkinson's disease. Behav Neurol 1995; 8:15-22.

18. Folstein MF, Folstein SE, McHugh PR. 'Mini mental state': a practical method for grading the cognitive state of patients for the clinician. J Psychiatr Res 1975; 12:189-98.

19. Fahn S, Elton RL, UPDRS Program Members. Unified Parkinson's Disease Rating Scale. In: Fahn S, Marsden CD, Goldstein M, Calne DB, eds. Recent Developments in Parkinson's Disease, Vol. 2. Florham Park, NJ: Macmillan Healthcare Information; 1987: 153-63, 293-304.

20. Pagonabarraga J, Kulisevsky J, Llebaria G, et al. Parkinson's disease-cognitive rating scale: a new scale specific for Parkinson's disease. Mov Disord 2008; 23:998-1005.

21. Gotham AM, Brown RG, Marsden CD. 'Frontal' cognitive function in patients with Parkinson's disease 'on' and 'off' levodopa. Brain 1988; $111(\mathrm{Pt}$ 2):299-321.

22. Leiva-Santana C, Alvarez-Saúco M. [Levodopa and cognitive disorders in Parkinson's disease]. Rev Neurol 2006; 43:95-100. Spanish.

23. Cools R, Barker RA, Sahakian BJ, Robbins TW. Enhanced or impaired cognitive function in Parkinson's disease as a function of dopaminergic medication and task demands. Cereb Cortex 2001; 11:1136-43.

24. Schneider JS, Sendek S, Yang C. Relationship between motor symptoms, cognition, and demographic characteristics in treated mild/moderate Parkinson's disease. PLoS One 2015; 10:e0123231.

25. Caparros-Lefebvre D, Pécheux N, Petit V, Duhamel A, Petit H. Which factors predict cognitive decline in Parkinson's disease? J Neurol Neurosurg Psychiatry 1995; 58:51-5. 